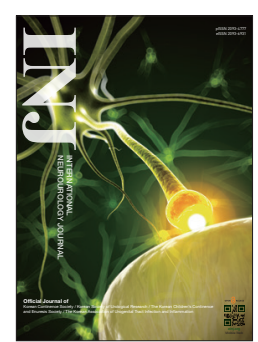

\title{
Application of Low-Cost, Easy-to-Use, Portable Biosensor Systems for Diagnosing Bladder Dysfunctions
}

\author{
Nosang V. Myung ${ }^{1}$, Sungyong Jung ${ }^{2}$, Jayoung Kim ${ }^{3,4,5,6}$ \\ ${ }^{1}$ Department of Chemical and Environmental Engineering, University of California Riverside, Riverside, CA, USA \\ ${ }^{2}$ Department of Electrical Engineering, University of Texas at Arlington, Arlington, TX, USA \\ ${ }^{3}$ Departments of Surgery and Biomedical Sciences, Cedars-Sinai Medical Center, Los Angeles, CA, USA \\ ${ }^{4}$ Samuel Oschin Comprehensive Cancer Institute, Cedars-Sinai Medical Center, Los Angeles, CA, USA \\ ${ }^{5}$ University of California Los Angeles, Los Angeles, CA, USA \\ ${ }^{6}$ Department of Urology, Gacheon University College of Medicine, Incheon, Korea
}

To the editor

A recently published series of articles in the International Neurourology Journal elegantly presented the current trends in Smart Health. In particular, 3 articles by Taeg-Keun Whangbo and colleagues [1,2] and Kang et al. [3] summarized how close we are to making personalized technology-based healthcare a reality in the clinical setting. Given that the authors provided an overview of the current applications of newly emerging technologies, we thought about how they could be utilized for diagnosing and monitoring nonfatal bladder diseases, such as interstitial cystitis (IC). Because of its unknown etiology and symptomatic manifestation as urinary discomfort in the absence of other causes, diagnosing IC is dependent on clinical parameters, such as pain, urinary urgency, and frequency. Additionally, due to the lack of proper conventional markers, diagnosis involves traditional urinalysis, urine culture, cystoscopy, bladder biopsy, and bladder hydro-distention. IC can cause a multitude of symptoms that have a negative impact on sexual activity and quality of life. The most significantly reported concern is chronic pelvic pain [4], which can also create sexual dysfunctions. Although the financial and personal burden of IC is extensive on the American public [5], there is still no clear diagnostic test for IC. Although our own group and several other laboratories have identified a series of IC urinary biomarkers, it is still unknown whether they would be clinically useful. Expanding use of this knowledge is costly and complexity of conventional analytic platforms, such as mass spectrometry, is hard to implement clinically.

Evaluating the impact duration, severity, pain localization, anxiety, and depression related to bladder dysfunctions may help identity risk factors. In addition to our own laboratory, many scientists have focused on developing biomarkers specific to each urological disease using biospecimens, such as tissue, blood, or other bodily fluids. Molecular biomarkers are widely accepted as a useful resource for prognoses and treatment response monitoring. Most importantly, accurate real-time monitoring of biomarkers may be immensely helpful for disease prevention, early diagnosing, and incidence rate reduction. Active oversight of biomarkers could be implemented using bio/ chemical sensors, which are analytical devices designed to detect biological or physiochemical markers, that can also be integrated with other smart health monitoring devices that sync wirelessly with smartphones. This data can be collected, stored, and analyzed in a shared cloud environment between patients and physicians. In order for such internet of biosensors to be successful in the clinical setting, they need to be portable, rapid, and cost-effective. For particularly efficient application in urology, effort should be focused on developing next generations of highly sensitive diagnostic biosensors that are portable, userfriendly, and low cost.
Corresponding author: Jayoung Kim (iD https://orcid.org/0000-0002-3683-4627 Departments of Surgery and Biomedical Sciences, Cedars-Sinai Medical Center, Davis 5071, 8700 Beverly Blvd., Los Angeles, CA 90048, USA

E-mail: Jayoung.Kim@cshs.org / Tel: +1-310-423-7168 / Fax: +1-310-967-3809 Submitted: January 21, 2019 / Accepted after revision: January 24, 2019
This is an Open Access article distributed under the terms of the Creative Commons Attribution Non-Commercial License (http://creativecommons.org/licenses/by-nc/4.0/) which permits unrestricted non-commercial use, distri-
bution, and reproduction in any medium, provided the original work is properly cited. 
Biosensors are analytical devices that can selectively detect and quantitatively measure analytes interacting with biological elements, such as tissues, microorganisms, antibodies, and nucleic acids. Biosensors consist of receptor-functionalized sensing materials that react to analytes and transducers that can convert biological responses into electrical signals. It is integrated with electronic circuits to readout and process signals, including amplifying, filtering, digitizing, analyzing, interfacing, transmitting, and displaying. The readout circuits are typically implemented with an operational amplifier, an analog-to-digital converter, a microcontroller, and a communication device. The types of biosensors can be categorized based on their detection mechanisms, such as electrochemical, thermal, electrical, magnetic, optical, and piezoelectric. Among these biosensors, electrochemical sensors are the most versatile, portable, and highly developed (approximately $58 \%$ of all chemical sensors) in the market. Prime examples of commercially available portable and easy-to-use electrochemical analyzers include glucose meters and portable blood analyzer (e.g., i-Stat Blood Chemistry Analyzer, Abbott, Princeton, NJ, USA), which have clearly demonstrated their ability to cost-effectively detect multiple analytes from small sample volumes. Like blood chemistry analyzers, high density electrochemical sensor arrays can be developed to accurately identify and quantify IC biomarkers from urine.

In addition to the development of portable analyzers for biological fluids, there has been rapid developments in gas sensor technology to mimic the human olfactory system to identify and quantify various gases (odors). This artificial olfactory system (e-nose) simulates the different stages of the human olfactory system, including sampling and filtering gaseous molecule. Using artificial gas sensors that odor molecules can react with, these sensors can then amplify and treat signal responses. Integrated artificial intelligence and web-based knowledge systems can evaluate key information to finally recognize the odor. Such devices could have a significant role in the early diagnosis and detection of IC by analyzing odors from urine samples.

Research and development related to mobile health devices will provide a game-changer in Smart Health and the future healthcare industry. Smart hospitals supported by computers, wireless network-based information, and communication technology are now rapidly evolving for more personalized care and electronic management of clinical records. As innovative thinkers, how can we apply biosensor technologies for medical diag- nostics and health monitoring, particularly in urological care?

- Conflict of Interest: No potential conflict of interest relevant to this article was reported.

\section{ACKNOWLEDGEMENTS}

This was supported by the Future Materials Discovery Program from the National Research Foundation of Korea (NRF). Funding was received from the Ministry of Science, ICT \& Future Planning (NRF-2016M3D1A1027836), and the NAVSEA METCAL program. The authors also acknowledge support from National Institutes of Health grants (1U01DK103260, 1R01DK100974, U24 DK097154, NIH NCATS UCLA CTSI UL1TR000124), Department of Defense grants (W81XWH-15-1-0415), Centers for Disease Controls and Prevention (1U01DP006079), the U.S.-Egypt Science and Technology Joint Fund by National Academies of Sciences, Engineering, and Medicine (NAS) and The United States Agency for International Development (USAID) (to J.K.). Any opinions, findings, conclusions, or recommendations expressed in this article are those of the authors alone, and do not necessarily reflect the views of USAID or NAS.

\section{REFERENCES}

1. Whangbo TK, Eun SJ, Jung EY, Park DK, Kim SJ, Kim CH, et al. Personalized urination activity recognition based on a recurrent neural network using smart band. Int Neurourol J 2018;22(Suppl 2):S91-100.

2. Eun SJ, Whangbo TK, Park DK, Kim KH. Development of personalized urination recognition technology using smart bands. Int Neurourol J 2017;21(Suppl 1):S76-83.

3. Kang M, Park E, Cho BH, Lee KS. Recent patient health monitoring platforms incorporating internet of things-enabled smart devices. Int Neurourol J 2018;22(Suppl 2):S76-82.

4. Katz L, Tripp DA, Carr LK, Mayer R, Moldwin RM, Nickel JC. Understanding pain and coping in women with interstitial cystitis/ bladder pain syndrome. BJU Int 2017;120:286-292.

5. Tung A, Hepp Z, Bansal A, Devine EB. Characterizing health care utilization, direct costs, and comorbidities associated with interstitial cystitis: a retrospective claims analysis. J Manag Care Spec Pharm 2017;23:474-82. 